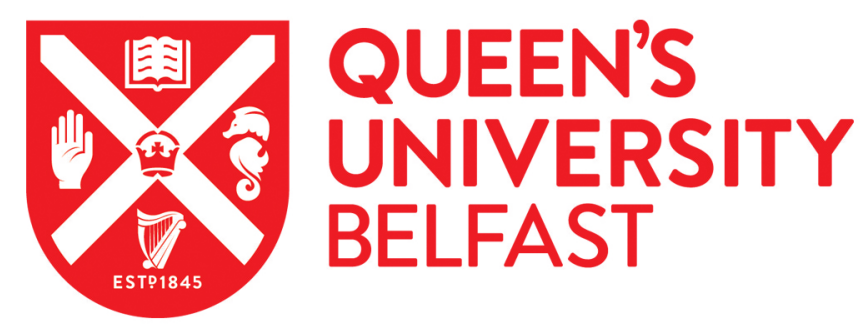

\title{
Development of a comprehensive analytical platform for the detection and quantitation of food fraud using a biomarker approach. The oregano adulteration case study
}

Wielogorska, E., Chevallier, O., Black, C., Galvin-King, P., Delêtre, M., Kelleher, C. T., Haughey, S. A., \& Elliott, C. T. (2018). Development of a comprehensive analytical platform for the detection and quantitation of food fraud using a biomarker approach. The oregano adulteration case study. Food Chemistry, 239, 32-39. https://doi.org/10.1016/j.foodchem.2017.06.083

\section{Published in:}

Food Chemistry

\section{Document Version:}

Peer reviewed version

Queen's University Belfast - Research Portal:

Link to publication record in Queen's University Belfast Research Portal

\author{
Publisher rights \\ Copyright 20 \\ This manuscript is distributed under a Creative Commons Attribution-NonCommercial-NoDerivs License \\ (https://creativecommons.org/licenses/by-nc-nd/4.0/), which permits distribution and reproduction for non-commercial purposes, provided the \\ author and source are cited.
}

\section{General rights}

Copyright for the publications made accessible via the Queen's University Belfast Research Portal is retained by the author(s) and / or other copyright owners and it is a condition of accessing these publications that users recognise and abide by the legal requirements associated with these rights.

Take down policy

The Research Portal is Queen's institutional repository that provides access to Queen's research output. Every effort has been made to ensure that content in the Research Portal does not infringe any person's rights, or applicable UK laws. If you discover content in the Research Portal that you believe breaches copyright or violates any law, please contact openaccess@qub.ac.uk. 
Chemistry

Elsevier Editorial system(tm) for Food

Manuscript Draft

Manuscript Number: FOODCHEM-D-16-06116R2

Title: Development of a comprehensive analytical platform for the detection and quantitation of food fraud using a biomarker approach. The oregano adulteration case study.

Article Type: Research Article (max 7,500 words)

Keywords: food, fraud, oregano, adulteration, mass spectrometry

Corresponding Author: Dr. Ewa Wielogorska,

Corresponding Author's Institution: QUB

First Author: Ewa Wielogorska

Order of Authors: Ewa Wielogorska; Olivier Chevallier, PhD; Connor Black; Pamela Galvin-King ; Marc Delêtre; Colin T Kelleher; Simon Haughey, PhD; Christopher Elliot, Prof.

Abstract: Due to increasing number of food fraud incidents, there is an inherent need for the development and implementation of analytical platforms enabling detection and quantitation of adulteration. In this study a set of unique biomarkers of commonly found adulterants became the targets in the development of a LC-MS/MS method which underwent a rigorous in-house validation. The method presented very high selectivity and specificity, excellent linearity (R2>0.988) low decision limits and detection capabilities (<2\%), acceptable accuracy (intra-assay 92-113\%, inter-assay 69-138\%) and precision ( $\mathrm{CV}<20 \%$ ). The method was compared with an established FTIR screening assay and revealed a good correlation of quali- and quantitative results (R2>0.81). An assessment of 54 suspected adulterated oregano samples revealed that almost 90\% of them contained at least one bulking agent, with a median level of adulteration of 50\%. Such innovative methodologies need to be established as routine testing procedures to detect and ultimately deter food fraud. 


\section{Development of a comprehensive analytical platform for the} 2 detection and quantitation of food fraud using a biomarker 3 approach. The oregano adulteration case study.

4

5 Ewa Wielogorska ${ }^{\mathrm{a}, *}$, Olivier Chevallier ${ }^{\mathrm{a}}$, Connor Black ${ }^{\mathrm{a}}$, Pamela Galvin-King ${ }^{\mathrm{a}}$, Marc 6 Delêtre $^{\mathrm{b}}$, Colin T. Kelleher ${ }^{\mathrm{b}}$, Simon A. Haughey ${ }^{\mathrm{a}}$, Christopher T. Elliot ${ }^{\mathrm{a}}$

7 a Institute for Global Food Security, Advanced ASSET Centre, School of Biological 8 Sciences, Queen's University Belfast, Northern Ireland, United Kingdom

$9{ }^{\mathrm{b}}$ DBN Plant Molecular Laboratory, National Botanic Gardens of Ireland, Glasnevin, Dublin 10 9, Ireland

*Corresponding author. Address: Institute for Global Food Security, Queen's University Belfast, 18-30 Malone Road, Belfast BT9 5BN, United Kingdom; e-mail: e.wielogorska@qub.ac.uk; phone number:+44(0)2890974155.

Remaining authors' e-mail addresses: Olivier Chevallier o.chevallier@qub.ac.uk; Connor Black cblack38@qub.ac.uk; Pamela Galvin-King p.galvin-king@qub.ac.uk; Marc Delêtre deletrem@tcd.ie; Colin T. Kelleher colin.kelleher@opw.ie; Simon A. Haughey s.a.haughey@qub.ac.uk; Christopher T. Elliott chris.elliott@qub.ac.uk;

Abstract:

Due to increasing number of food fraud incidents, there is an inherent need for the development and implementation of analytical platforms enabling detection and quantitation of adulteration. In this study a set of unique biomarkers of commonly found adulterants became the targets in the development of a LC-MS/MS method which underwent a rigorous in-house validation. The method presented very high selectivity and specificity, excellent linearity $\left(\mathrm{R}^{2}>0.988\right)$ low decision limits and detection capabilities $(<2 \%)$, acceptable accuracy (intra-assay 92-113\%, inter-assay 69-138\%) and precision $(\mathrm{CV}<20 \%)$. The method was compared with an established FTIR screening assay and revealed a good correlation of quali- and quantitative results $\left(\mathrm{R}^{2}>0.81\right)$. An assessment of 54 suspected adulterated oregano 
30 samples revealed that almost $90 \%$ of them contained at least one bulking agent, with a

31 median level of adulteration of $50 \%$. Such innovative methodologies need to be established 32 as routine testing procedures to detect and ultimately deter food fraud.

33 Keywords: food, fraud, oregano, adulteration, mass spectrometry

34

35

36

37

38

39

40

41

42

43

44

45

46

47

48

49

50

51

52

53

54 
In 2002 the European Union recognised the necessity for introducing the Food Law (European Food Safety Authority, 2002) to protect consumers' health and provide improved confidence that the EU, as a major global trader in food, has the most stringent food safety laws in the world. In order to facilitate consumers' informed choices, 'the food law should aim at the prevention of fraudulent or deceptive practices; the adulteration of food; and any other practices which may mislead the consumer' (European Food Safety Authority, 2002). Nevertheless, to date EU legislation does not provide a definition of food fraud. In a European Parliament report 2013/2091(INI), following the horsemeat scandal, the key characteristics of food fraud were outlined and were similar to the definitions already introduced in the USA (Johnson, 2014) - i.e. non-compliance with food law and/or misleading the customer which is done intentionally and for financial gain resulting from fraudulent practices such as adulteration, substitution, tampering or counterfeiting. At the same time the 'Food Fraud Network' started to collect and exchange information in order to detect and prevent food fraud in the EU (European Commission, 2016). The largest available summary of records (over 2,000) on food fraud is contained within the U.S. Pharmacopeia Food Fraud Database (U.S. Pharmacopeial Convention, 2016), which encompasses both scholarly and media reports on the subject area. According to this database, during years 2011-2012 the number of fraud records increased by 60\% when compared with the period spanning 1980-2010. The first and second most affected food ingredients quoted being oils and spices respectively, with the latter accounting for 16\% of all records (Johnson, 2014). Amongst these, there have been number of recent fraud incidents, potentially posing a health risk, such as addition of toxic colorants or botanic substitutes (Moore, Spink, \& Lipp, 2012; U.S. Pharmacopeial Convention, 2016). In addition there have also been reports on bulking agents used for the purpose of substitution/dilution of herbs (U.S. Pharmacopeial Convention, 2016; Marieschi, Torelli, Bianchi, \& Bruni, 2011; Marieschi, Torelli, Poli, Bianchi, \& Bruni, 2010) which is in agreement with a recent study focusing on oregano adulteration (Black, Haughey, Chevallier, Galvin-King, \& Elliott, 2016). Almost 25\% of tested UK oregano samples $(n=78)$ were found to be adulterated with bulking agents, most commonly olive and myrtle leaves. The levels of adulteration in some samples was found to be over $70 \%$. This example of adulteration is a classic case of food fraud where the aim is to mislead consumers for purely financial gain. This type of adulteration may pose health threats due to unknown 
status of the bulking agents, thus exposing consumers to potentially toxic compounds present in the botanical adulterants employed as well as potential microbiological and/or chemical contaminants.

Each food fraud incident has the potential to threaten consumers' well-being but also undermine confidence in the EU food market in which the herbs and spices' share is worth 1.8 billion Euro in the EU and 2.97 billion dollars world-wide (Marieschi, Torelli, Poli, Sacchetti, \& Bruni, 2009). Thus, the European Parliament issued a call to develop and implement technologies and methods to detect food fraud including sensor technology and fingerprinting approach (European Parliament, 2013). Such methods, including FourierTransform Infrared spectroscopy (FTIR) have been already successfully implemented in food fraud detection (Ellis, Muhamadali, Haughey, Elliott, \& Goodacre, 2015). DNA sequencing techniques are also considered to be reliable, nevertheless, their limitations have been discussed (Parveen, Gafner, Techen, Murch, \& Khan, 2016) and make them unsuitable to be employed as stand-alone tools in the field of authentication. Furthermore, according to European legislation concerning the performance of analytical methods (European Commission, 2002), those approaches would be classified as screening methods and as such they are not fit for the purpose of confirming adulteration to required legal standards, due to lack of chemical structure confirmation, if the legal action is to be pursued by industry or regulators. High Resolution Mass Spectrometry (HRMS) based fingerprinting approaches have also been employed in food authentication, however even though such methods provide the possibility of detecting unusual deviations within the sample set, instrumental analysis is time consuming, requires expensive equipment, data storage facilities and high processing power (Esslinger, Riedl, \& Fauhl-Hassek, 2014). Available targeted mass spectrometry based methods designed for the purpose of adulteration confirmation usually employ food profiling approach whereby sample classification is based on analysing a selected group of matrix constituents such as flavonoids in Ginkgo biloba or ginsenosides in Panax Ginseng (Xie, Chen, Liang, Wang, Tian, \& Upton, 2006; Yuan, Wang, Chen, Ye, \& Zhou, 2016). Such validated analytical methods ensure accuracy of the analytical determination (Esslinger, Riedl, \& Fauhl-Hassek, 2014), however are prone to 'targeted designed adulteration' (Sanzini, Badea, Dos Santos, Restani, \& Sievers, 2011) due to increased knowledge regarding chemical composition of food commodities.

Consequently, designing an analytical approach suitable for rapid, cheap and reliable detection and confirmation of adulteration still presents a challenge. Many important issues 
such as selectivity and specificity of chosen markers in the presence of various botanic adulterants, methods' ruggedness as well as inherent biodiversity (De Falco, Mancini, Roscigno, Mignola, Taglialatela-Scafati, \& Senatore, 2013) must all be considered during experimental design. Thus, the aim of the present study was to explore the possibility of designing a holistic system allowing for both fast and reliable FTIR screening as well as cost effective, simplified, liquid chromatography tandem - mass spectrometry (LC-MS/MS) based confirmation for the purpose of oregano adulteration detection and quantitation. The successful outcome of this study could lead to many other future applications of such a system for the analysis of other types of food fraud.

\section{Materials and methods}

\subsection{Reference plant material and identity confirmation}

A set of 38 samples were employed in the current LC-MS/MS method development and validation. Those included either certified reference materials (Origanum vulgare leaf and Olea europaea leaf referenced against vouchered (AH0024) and reagent grade biomass reference material respectively, both from LGC standards, Teddington, UK) or samples supplied with full provenance and traceability including leaves of culinary Origanum species such as Origanum vulgare and Origanum onites $(\mathrm{n}=11)$ as well as samples of potential bulking agents including olive leaves - Olea europaea subsp. europaea $(\mathrm{n}=6)$, Myrtaceae leaves including Myrtus communis and Myrtus communis var. communis $(\mathrm{n}=6)$, phlomis leaves - Phlomis $x$ cytherea $(\mathrm{n}=2)$, sumac leaves - Rhus coriaria $(\mathrm{n}=3)$, hazelnut leaves Corylus avellana $(\mathrm{n}=3)$, sage leaves - Salvia officinalis $(\mathrm{n}=3)$ and cistus leaves - Cistus creticus $(\mathrm{n}=2)$. In order to experimentally confirm the identification of the samples, specimens were compared morphologically against herbarium specimens and were also assessed using DNA barcoding. Material has been deposited into the DBN herbarium tissue bank in the National Botanic Gardens of Ireland (registration number DBN2017:04).

\subsubsection{DNA extraction and PCR conditions}

Genomic DNA was extracted from approximately $20 \mathrm{mg}$ of lyophilized leaf tissue using Macherey-Nagel NucleoSpin ${ }^{\circledR}$ Plant II kits according to the manufacturer's instructions, with 
the exception that the cell lysis incubation step at $65^{\circ} \mathrm{C}$ was extended to $45 \mathrm{~min}$. Species identity was confirmed using DNA barcoding. Four regions were used, three plastid and one nuclear region. The plastid regions were trnH-psbA (Kress, Wurdack, Zimmer, Weigt, \& Janzen, 2005), trnL-trnF (Taberlet, Coissac, Pompanon, Gielly, Miquel, Valentini, et al., 2007) and matK (Yu, Xue \& Zhou, 2011). The nuclear region was the Internal Transcribed Spacer region (ITS) (Kress, Wurdack, Zimmer, Weigt, \& Janzen, 2005). Polymerase chain reactions (PCR) contained $1 \mu \mathrm{L}(\sim 50 \mathrm{ng})$ of DNA template, $1 \times$ Bioline MyTaq $^{\mathrm{TM}}$ Red Mix (Bioline, USA) and $0.5 \mu \mathrm{M}$ of the forward and reverse primers (Eurofins, Germany) in a total volume of $20 \mu \mathrm{L}$. PCR cycle conditions were: $94^{\circ} \mathrm{C}$ for $2 \mathrm{~min}$ followed by 40 cycles of $94^{\circ} \mathrm{C}$ for $1 \mathrm{~min}, 58^{\circ} \mathrm{C}$ for $1 \mathrm{~min}$ and $72^{\circ} \mathrm{C}$ for $1 \mathrm{~min}$, with a final $10 \mathrm{~min}$ extension at $72^{\circ} \mathrm{C}$. PCR products were purified using the ExoSAP method (New England Biolabs) and sent to Macrogen Europe for direct sequencing in one direction.

\subsubsection{DNA sequence analysis}

DNA sequences were aligned and cleaned using Sequencher v. 4.10.1 (Gene Codes Corporation). Cleaned sequences were BLASTed against sequences available in GenBank and the top hits were checked for matches with the expected taxon. Results are presented in Table S1.

\subsection{Sample preparation}

The protocol described was based on the extraction published in the previous study (Black, Haughey, Chevallier, Galvin-King, \& Elliott, 2016) and simplified herein by decreasing the volume of the extraction solvent from $2 \mathrm{~mL}$ to $0.5 \mathrm{~mL}$, sonication time was shortened from $15 \mathrm{~min}$ to $10 \mathrm{~min}$ while final solvent exchange and filtration steps were abandoned to shorten the analysis time. Shortly, the samples were milled to a homogenous powder on a PM-100 Retsch Planetary Ball Mill (Retsch, Haan, Germany). $50 \mathrm{mg}(+/-1 \%)$ was weighed out on a Discovery DV215CD Analytical Balance (Ohaus Europe GmbH, Nanikon, Switzerland) into a $1.5 \mathrm{~mL}$ Eppendorf tube and $500 \mu \mathrm{L}$ of ultra-pure water $(18.2 \mathrm{M} \Omega / \mathrm{cm}) / \mathrm{LC}-\mathrm{MS}$ Chromasolv methanol (1:1, v/v) solution added (Merck Millipore, Billerica, USA and Sigma-Aldrich, St Louis, MO, USA respectively). Samples were then extracted by vortexing $10 \mathrm{~min}$ at 2,500 rpm with a DVX-2500 Multitube Vortexer (VWR International, Lutterworth, UK), sonicated for 10 min in camSonix C1274 sonicator (Camlab, Cambridge, UK) and centrifuged at 9,500 $\mathrm{x} g$ in a MIKRO 200R centrifuge (Hettich UK, Salford, UK). $300 \mu \mathrm{L}$ of the resulting 
supernatant was transferred into a maximum recovery vial (Waters, Manchester, UK) and analysed on the same day.

\subsection{Untargeted LC-HRMS analysis}

An untargeted approach has been developed to detect herb adulteration and is the basis of present, follow up targeted study (Black, Haughey, Chevallier, Galvin-King, \& Elliott, 2016). Briefly, oregano and adulterants' (olive, Myrtaceae, phlomis, sumac, and hazelnut leaves) extracts were analysed employing Waters Acquity I-Class UHPLC. The LC column and mobile phases system consisted of Waters Acquity HSS T3 column $(2.1$ x 100 mm, $1.8 \mu \mathrm{m})$, maintained at $45^{\circ} \mathrm{C}$ while the pump was operated at a flow rate of $0.4 \mathrm{~mL} / \mathrm{min}$ with mobile phases A, $0.1 \%$ formic acid in water and B, $0.1 \%$ formic acid in methanol. A linear gradient from $99 \% \mathrm{~A}$ to $1 \% \mathrm{~A}$ over $20 \mathrm{~min}$ was applied and the same LC conditions were applied during initial targeted method development. Mass spectral data were acquired over the range $\mathrm{m} / \mathrm{z}$ 50-1200 in both positive and negative ion mode using a Waters Acquity I-Class UHPLC coupled to a G2-S quadrupole time of flight (Q-ToF) mass spectrometer. The raw data generated were processed following a Progenesis QI 2.0 software work flow. The processed data were then subjected to multivariate analysis using SIMCA 14.0 (Umetrics, Sweden). Principal component analysis (PCA) and orthogonal partial least squares discriminate analysis (OPLS-DA) were used for building the qualitative models.

The PCA score plots generated (Figure S1 - supplementary material) showed clear separation between the authentic oregano and adulterant samples. The discovery of characteristic biomarkers for each adulterant was achieved by generating individual OPLS-DA models for each adulterant against pure oregano, with Table S2 (supplementary material) identifying the measure of fit $\left(\mathrm{R}^{2}\right)$, the measure of predicative ability $\left(\mathrm{Q}^{2}\right)$ and $\mathrm{RMSECV}$ values for each OPLS-DA model. These individual models enabled us to create S-plots which identified a set of ions in both ionisation modes for each adulterant. As a result, these ions were thoroughly investigated using both the raw data and Progenesis QI 2.0 to guarantee that they were not present in any of the authentic oregano samples. Overall a total of 23 adulterant markers were identified and were used to generate an in-house database within Progenesis QI software. Due to lack of full chemical identification all biomarkers were assigned arbitrary IDs stemming from the first letter of the adulterant's name, consecutive number and ionisation mode in 
which they were analysed. Tables with all biomarkers' arbitrary IDs, their retention times and neutral masses are presented in supplementary material (Table S3 and S4).

\subsection{Targeted LC-MS/MS analysis}

\subsubsection{Experimental conditions}

Analysis was performed on an Acquity UHPLC I-Class system (Waters, Milford, MA, USA) coupled to Xevo TQ-MS triple quadrupole mass analyser (Waters, Manchester, UK) operating both in positive and negative electrospray ionisation modes. The following settings were applied: capillary voltage was set at 2.5 and 1.0 for positive and negative mode respectively. The desolvation and source temperatures were set at 500 and $150^{\circ} \mathrm{C}$, respectively, while nitrogen cone and desolvation flow rates were set to 50 and $750 \mathrm{~L} / \mathrm{hr}$. Argon was employed as a collision gas, with a flow of $0.15 \mathrm{~mL} / \mathrm{min}$, yielding a collision cell pressure of $2.5 \times 10^{-3} \mathrm{mBar}$. Inter -scan and -channel delays were both set to $3 \mathrm{~ms}$ while dwell times ranged from 14 to $76 \mathrm{~ms}$.

Analytes' separation was performed on a Waters Acquity HSS T3 column $(2.1 \times 100 \mathrm{~mm}, 1.8$ $\mu \mathrm{m}$ ), maintained at $45^{\circ} \mathrm{C}$ while the pump was operated at a flow rate of $0.4 \mathrm{~mL} / \mathrm{min}$. Mobile phases consisted of $\mathrm{A}, 0.1 \%$ formic acid in water and $\mathrm{B}, 0.1 \%$ formic acid in methanol. A linear gradient was then adjusted to achieve more efficient separation time i.e. isocratic $0-$ $0.8 \min 65 \% \mathrm{~A}$, linear $0.8-4.20 \mathrm{~min} 20 \% \mathrm{~A}$ and isocratic column equilibration $4.30-6 \mathrm{~min}$ at $65 \%$ A. After each injection the needle was washed and purged with water/methanol $(1: 1$, $\mathrm{v} / \mathrm{v})$ solution.

\subsubsection{Biomarker selection and method development}

Monitored biomarkers' transitions were determined during multivariate analysis of data acquired during untargeted UHPLC-HRMS analysis. Each biomarker's spectrum was acquired on the TQ-MS in full scan mode applying the same chromatographic conditions (described in 2.3.) as in the non-targeted analysis and compared to respective spectrum acquired on Q-ToF (supplementary material - Figure S4 - S8). At least three most prominent fragments of each biomarker's precursor were selected for its respective MRM window and 
chromatographic conditions adjusted, substantially decreasing analysis time. The most suitable spectrometric conditions were achieved by repeated on-column injections of a pooled adulterant sample. Each of the selected adulterants was found to contain at least one specific molecule (specified in Figure 1) i.e. olive leaves (O1- and O2-), Myrtaceae leaves (M1- and M1), sumac leaves (S2 and S3), hazel leaves (H1, H2 and H1-) and phlomis leaves (Ph2-). Unfortunately, no fully specific marker for oregano itself could have been established. To date none of the selected compounds has been successfully identified due to high number of structural isomers of suspected compounds, nevertheless, the minimal requirements of chemical characterization of unknown metabolites is fulfilled as outlined by Chemical Analysis Working Group within Metabolomics Standards Initiative (Sumner, Amberg, Barrett, Beale, Beger, Daykin, et al., 2007). To increase reliability of the method, the three most selective and specific fragments for each of the biomarkers were identified during selectivity study (Figure 1), yielding 5.5 identification points per compound as defined in Commission Decision 2002/657/EC (European Commission, 2002), and their ion ratios closely monitored.

\subsubsection{Method validation}

The method was validated based on Commission Decision 2002/657/EC (European Commission, 2002).

Selectivity and specificity were established by assessing interferences in the UHPLC-MS/MS traces between a number of authenticated samples' extracts ( $\mathrm{n}=38$, as described in 2.1.) representing different varieties of oregano, bulking agents and other culinary herbs, including cistus and sage which have not been previously assessed by HRMS, thus no markers could be elucidated.

Linearity was assessed for two main adulterants i.e. olive and Myrtaceae leaves as assessed previously during commercial samples' survey (Black, Haughey, Chevallier, Galvin-King, \& Elliott, 2016). Four different calibration curves per adulterant were prepared, using authenticated samples with most varied concentrations of selected biomarkers (as assessed during pre-screening) at levels 5, 10, 20, 40 and $60 \%(w / w)$ adulteration each.

Due to the fact the method was developed employing incurred material which composition varies and is dependent on factors such as genotype, environment or developmental stage (De 
276 Falco, Mancini, Roscigno, Mignola, Taglialatela-Scafati, \& Senatore, 2013), the assessment 277 of resulting biodiversity within species (United Nations, 1992) in different oregano and 278 adulterant matrices $(\mathrm{n}=21)$ was performed by analysing composites of varying oregano and 279 either of the two main adulterants samples at adulteration level of $10 \%(\mathrm{w} / \mathrm{w})$. Results of this 280 study were also employed in the intra-day repeatability assessment.

281 To assess matrix effects as well as their influence on accuracy and within laboratory reproducibility (WLR), analysis of 3 levels of adulteration with 3 mixtures containing both olive and Myrtaceae leaves was performed. Mix 1 consisted of $10 \%$ and $60 \%$; mix $2-30 \%$ and 30\%; mix $3-60 \%$ and 10\% (w/w) of olive and Myrtaceae leaves. Six replicates of each mix were extracted and analysed by three different operators on three different days $(n=54)$.

Decision limit $(\mathrm{CC} \alpha)$ and detection capability $(\mathrm{CC} \beta)$ - as defined in (European Commission, 2002) - were established by performing a linear regression employing WLR results. The intercept's standard deviation was used for establishment of limits. To confirm the extrapolation results, the standard deviation of measured adulteration of 20 extracts, blended at $1.5 \%(\mathrm{w} / \mathrm{w})$ adulteration level (composite sample containing all five adulterants), was used to experimentally confirm those limits for both olive and Myrtaceae leaves and assess them for the rest of the adulterants.

\subsection{Screening employing Fourier-Transform Infrared (FTIR) analysis}

\subsubsection{Sample preparation of adulterated oregano}

Adulterated samples were prepared by spiking authentic oregano samples in $10 \%$ increments, from $0-100 \% \mathrm{w} / \mathrm{w}$, with all adulterants. This range was chosen as economically motivated adulteration tends to occur at concentrations at least $10 \%$ and above.

\subsubsection{Spectral Data Acquisition using FTIR}

For FTIR, the milled samples were placed in the ATR sample area of a Thermo Nicolet iS5 spectrometer (Thermo Fisher Scientific, Dublin, Ireland) equipped with ATR iD5 diamond crystal and ZnSe lens and DTGS KBr detector and acquired as previously described (Black, Haughey, Chevallier, Galvin-King, \& Elliott, 2016). 
307

308

309

310

311

312

313

314

315

316

317

318

319

320

321

322

323

324

325

326

327

328

329

330

331

332

333

334

335

336

337

Multivariate analysis was used for quantitative analysis FTIR spectroscopic data. The analysis was carried out using the chemometric software packages TQ Analyst (Thermo Fisher Scientific, Dublin, Ireland) using the partial least squares regression (PLS-R) algorithm in the current study for quantitative model building as well as SIMCA 14 (MKS, Sweden) for qualitative model building using principal component analysis (PCA). The quantitative calibration model selected was obtained using the lowest standard error of prediction to minimise the possibility of over-fitting when the model accuracy was evaluated. Calibration models were developed from FTIR spectral data in the wavenumber range 600$4000 \mathrm{~cm}^{-1}$ which included the molecular fingerprint region. The data pre-treatments examined were none (raw data), 1st and 2nd derivatives calculated in conjunction with the SavitzkyGolay smoothing methods and also pre-processed using the standard normal variate (SNV) technique which compensates for differences in pathlengths due to light scattering effects. To select the calibration and validation data, TQ Analyst uses the information in the components table, related to the analyte of interest, to construct a multidimensional sample space. It then uses statistical guidelines related to the spectral and/or concentration data to randomly select calibration and validation standards from the space sample model which is repeated until a complete set of each is available.

\section{Results and discussion}

Selectivity and specificity of the LC-MS/MS method were assessed by monitoring chromatographic traces after injecting different extracts of oregano and the most common adulterants - olive leaves and Myrtaceae leaves as well as less common bulking agents such as sumac, hazelnut, phlomis and cistus or other culinary herbs for potential interferences such as sage $(n=38)$. No cross-talk was noted for the selected biomarkers. To further increase reliability, confirmation of adulteration with a specific bulking agent was only permitted in the presence of all associated biomarkers. Oleuropein, which was previously described in the literature as a marker selective for oregano adulteration with olive leaves (Bononi \& Tateo, 2011), was shown to be also present in Myrtaceae leaves (Figure S9 - supplementary material), which is not reported in the literature, and was thus rejected in the present study. Nevertheless, the efficiency of electrospray ionisation varies depending on the analytes' 
chemical structure and lack of analytical standards for other olive leaves markers halts reliable assessment of resulting differences in sensitivity. Thus, the possibility of oleuropein presence in the samples due to even minimal sample contamination with olive leaves, which would not be picked up during DNA analysis nor FTIR, should not be excluded and further examination of Myrtaceae leaves for the presence of oleuropein should be performed.

Linearity assessment revealed that only one of two selected biomarkers specific to each olive and Myrtaceae leaves expressed acceptable linear correlation with the level of adulteration $(\mathrm{O} 2-$ and $\mathrm{M} 1$, as presented in Figure S2). Employing those biomarkers yielded excellent linearity in varying matrices $(n=4)$ with goodness of fit coefficients higher than 0.988 for six point curves (including zero) for both adulterants tested. Biodiversity assessment (Table 1) revealed that even in varying matrices, the accuracy of method is acceptable, ranging from $109-113 \%$ for olive and $100-110 \%$ for Myrtaceae leaves. Good intra-day repeatability was also achieved in this study with maximum covariance of 17 and 18\% for olive and Myrtaceae leaves. The assessed repeatability, thus accounts for natural variability of the matrix constituents, markers' levels in the assessed matrices, as well as possible commercial sample composition and inherent analytical within-day variability of the method. Nevertheless, samples containing biomarkers at levels outside characterised range should not be ruled out.

During the WRL assessment (Table 2), the method presented excellent reproducibility with covariance values below 10\%. Nevertheless, accuracy of the method was shown to be affected by the presence of other adulterants at levels higher than $30 \%$. Samples with $10 \%$ adulteration were heavily affected by the presence of the other adulterant, at $60 \%$, revealing signal enhanced by almost $40 \%$ in case of the olive leaves biomarker while the Myrtaceae leaves biomarker was suppressed by $30 \%$. Such interactions were not noted whenever two adulterants were tested separately during the biodiversity study, thus alteration in accuracy was attributed to matrix effects exerted by the other adulterant and was shown to be heavily dependent on its concentration. Thus, attention must be paid whenever quantifying samples adulterated with other bulking agents at levels higher than $30 \%$. Nonetheless, a performed regression presented excellent linearity (Figure S3) and yielded very low values of CC $\alpha$ $1.27 \%$ and $1.18 \%$ - CC $\beta-2.16 \%$ and 2.02\% - for olive and Myrtaceae leaves, respectively (Table 2). To confirm those results, 20 oregano samples were adulterated at the level of $1.5 \%$ (chromatographic traces presented in Figure S10 - supplementary material) and measured concentration deviation assessed to determine experimental CC $\beta$ values $-1.76 \%$ and $1.54 \%$ which were both similar to those achieved through extrapolation for olive and Myrtaceae 
leaves. The values may be slightly lower due to the assessment performed at a singular adulteration level and on the same day, thus limiting experimental variability. Additionally, $\mathrm{CC} \beta \mathrm{s}$ were assessed for three other bulking agents i.e. sumac, hazelnut and phlomis leaves. Assessed standard deviation, based on the absolute response, was employed and following detection capabilities established: $1.45 \%, 1.43 \%, 1.70 \%$ for sumac, hazelnut and phlomis leaves respectively. The achieved limits are in line with 'oregano cleanliness specifications' outlined by the American Spice Trade Association and European Spice Association i.e. 1\% and 2\% (w/w) of extraneous matter respectively (American Spice Trade Association, 1999; European Spice Association, 2015).

Regarding FTIR analysis, Figure 2 presents the PCA qualitative model and the PLS-R quantitative model for both Origanum onites and vulgare adulterated with olive leaves and Myrtaceae leaves. The PCA models, unsupervised and based purely on the differences found in the spectral data, shows clearly the differences between samples spiked at various levels of adulterants. In both PCA models for onites and vulgare respectively, the first three principal components calculated explain the majority of the differences $(92.5 \%$ and $93.7 \%)$ between samples: onites PC1 55.6\% PC2 30.8\% PC3 6\%; vulgare PC1 57.3\% PC2 30.5\% PC3 5.9\%. Using the unsupervised PCA technique, clear differences can be observed between the adulterated and authentic oregano samples. The PLS-R calibration models were derived using pre-processing (SNV, 1st derivative with Savitzsky-Golay smoothing) of the FTIR spectral data and includes the corresponding root mean squared error of calibration (RMSEC) and root mean squared error of prediction (RMSEP). These latter values give an indication of the quality of the calibration models generated. The calibration models for each set of samples gave excellent correlation values $\left(\mathrm{R}^{2}\right)$ of 0.99 whilst the corresponding values for RMSEC and RMSEP were found to be $1.48-1.68 \%$ and $1.42-2.64 \%$. The graphical representation for each of the PLS calibration models generated for respective oregano species show the actual concentration on $\mathrm{X}$-axis versus calculated (predicted) concentration on the $\mathrm{Y}$-axis. The samples used for calibration are indicated by the circles whilst those used for validation are indicated by crosses. The calibration models produced with subsequent validation data indicate that this FTIR spectral data approach could potentially be used to detect fraud and adulteration of oregano with an indication of the levels of adulterants present. Therefore routine testing of oregano shipments with FTIR and chemometrics could potentially lead to reduced levels of fraud in the herb sector. 
405 Both methods described were compared by means of qualitative and quantitative analysis of 54 , suspect, oregano samples.

407 Qualitative correlation of methods for adulteration determination (defined as correct classification of each of the 54 samples as adulterated or pure) for both olive leaves and Myrtaceae were $94 \%$, due to 3 adulterated samples being below the FTIR's model lowest concentration (10\%). Additionally, out of 9 samples containing sumac, 6 were below the FTIR's lowest concentration thus misclassified, but still yielded an $89 \%$ correct classification rate. None of the samples were found to be adulterated with hazelnut or phlomis in the study. However, 12 samples were adulterated with cistus which were detected by FTIR only, as no exclusive biomarker could be found to confirm cistus adulteration by LC-MS/MS.

In terms of quantitative correlation for olive and Myrtaceae leaves adulteration, both methods show good correlation (Figure 3). In the case of samples with high levels of adulteration with olive leaves (4 samples above 90\%) the methods showed slightly lower correlation $\left(\mathrm{R}^{2}=\right.$ 0.73), however, if only samples with adulteration levels below $90 \%$ are taken into consideration, higher correlation was achieved $\left(\mathrm{R}^{2}=0.85\right)$. Keeping that limitation in mind, the estimation of the levels of adulteration for both olive and Myrtaceae leaves $\left(\mathrm{R}^{2}=0.81\right)$ achieved during FTIR screening can be relied on, due to almost neutral slope and intercept values achieved during correlation assessment (Figure 3).

The samples chosen for LC-MS/MS analysis were initially suspected of adulteration via the FTIR method as well as a portion of samples shown to be non-adulterated. The results have shown that the samples analysed were correctly predicted by each method with $87 \%$ of samples containing at least one of the bulking agent tested for (Figure 4a). Out of those, $42 \%$ contained more than one adulterant. The median level of adulteration was established to be $50 \%\left(25^{\text {th }}\right.$ percentile at $31 \%$ and $75^{\text {th }}$ percentile at $\left.75 \%\right)$ (Figure $\left.4 \mathrm{~b}\right)$.

4. Conclusions

The newly developed platform consisting of a screening FTIR assay and an LC-MS/MS confirmatory, semi-quantitative assay for detection, confirmation and quantitation of oregano 433 adulteration was thoroughly assessed. Total analysis time, including sample preparation, was 43430 min, with minimal sample processing costs required. The LC-MS/MS method presented 
high selectivity and specificity, good repeatability and accuracy, with low limits of detection and quantitation, in line with the herb and spice industry guidelines. Validation study underlines the need of complex assessment of the tested matrices, expected adulterants and their mixtures whenever developing similar methods due to possible matrix effects, directly influencing the quantitation obtained. Even though the platform developed presented good analytical performance in detecting selected non-culinary bulking agents, oregano samples have been also reported to be substituted with other, volatile species such as marjoram and winter savory which highlights the need for further method development. Also, fraudsters are known to change their mode of action when exposed, possibly leading to abandoning the bulking agents employed which stresses the importance of further expansion and improvement of botanicals data base to increase both methods' applicability and ruggedness. The results also stress the importance of adopting a holistic approach to the detection of adulteration. Auxiliary methods, such as DNA barcoding or FTIR in this case, may reveal invaluable insights into tested sample's composition and improve their classification due to advantages of multi-variate, fingerprint analysis.

The results obtained in terms of the level and complexity of oregano adulteration clearly demonstrate a need for further development of such analytical platforms due to the increasing likelihood of adulteration for economic gain, now being discovered in many food products.

(1)

(1)

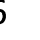

7

8

\section{Acknowledgements}

The research undertaken was funded by a UK BBSRC CASE studentship BB/M0503162/1. 
American Spice Trade Association (1999). American Spice Trade Association's Cleanliness specifications for Spices, Seeds and Herbs. http://www.astaspice.org/food-safety/cleanlinessspecifications/ Accessed 05.10.16

Black, C., Haughey, S. A., Chevallier, O. P., Galvin-King, P., \& Elliott, C. T. (2016). A comprehensive strategy to detect the fraudulent adulteration of herbs: The oregano approach. Food Chemistry, 210, 551-557.

Bononi, M., \& Tateo, F. (2011). Lc-Esi-Ms/Ms Identification of Oleuropein as Marker of Olea Europaea L. Leaves Used as a Bulking Agent in Ground Oregano and Sage. Italian Journal of Food Science, 23(3), 245-251.

De Falco, E., Mancini, E., Roscigno, G., Mignola, E., Taglialatela-Scafati, O., \& Senatore, F. (2013). Chemical Composition and Biological Activity of Essential Oils of Origanum vulgare L. subsp vulgare L. under Different Growth Conditions. Molecules, 18(12), 14948-14960.

European Commission (2002). Commission Decision of 12 August 2002 implementing Council Directive 96/23/EC concerning the performance of analytical methods and the interpretation of results (2002/657/EC). Official Journal of the European Communities, L 221/8-36.

$\begin{array}{lllll}\text { European } & \text { Commission } & \text { (2016). } & \text { Food } & \text { Fraud. }\end{array}$ http://ec.europa.eu/food/safety/official_controls/food_fraud/index_en.htm Accessed 08.10.16

European Food Safety Authority (2002). Regulation (EC) No 178/2002 of the European Parliament and of the Council of 28 January 2002 laying down the general principles and requirements of food law, establishing the European Food Safety Authority and laying down procedures in matters of food safety. Official Journal of the European Communities, L 31/1-24.

Ellis, D. I., Muhamadali, H., Haughey, S. A., Elliott, C. T., \& Goodacre, R. (2015). Point-and-shoot: rapid quantitative detection methods for on-site food fraud analysis - moving out of the laboratory and into the food supply chain. Analytical Methods, 7(22), 9401-9414.

European Parliament (2013). EP Report 2013/2091(INI). Report on the food crisis, fraud in the food chain and the control thereof. European Parliament - Committee on the Environment, Public Health and Food Safety

European Spice Association (2015). European Spice Association Quality Minima Document. http://www.esa-spices.org/index-esa.html/publications-esa Accessed 05.10.16

Esslinger, S., Riedl, J., \& Fauhl-Hassek, C. (2014). Potential and limitations of non-targeted fingerprinting for authentication of food in official control. Food Research International, 60, 189-204.

Johnson, R. (2014). Food Fraud and "Economically Motivated Adulteration" of Food and Food Ingredients. DC: Congressional Research Service.

Kress, W. J., Wurdack, K. J., Zimmer, E. A., Weigt, L. A., \& Janzen, D. H. (2005b). Use of DNA barcodes to identify flowering plants. Proceedings of the National Academy of Sciences of the United States of America, 102(23), 8369-8374.

Marieschi, M., Torelli, A., Poli, F., Sacchetti, G., \& Bruni, R. (2009). RAPD-Based Method for the Quality Control of Mediterranean Oregano and Its Contribution to Pharmacognostic Techniques. Journal of Agricultural and Food Chemistry, 57(5), 1835-1840.

Marieschi, M., Torelli, A., Poli, F., Bianchi, A., \& Bruni, R. (2010). Quality control of commercial Mediterranean oregano: Development of SCAR markers for the detection of the adulterants Cistus incanus L., Rubus caesius L. and Rhus coriaria L. Food Control, 21(7), 998-1003.

Marieschi, M., Torelli, A., Bianchi, A., \& Bruni, R. (2011). Detecting Satureja montana L. and Origanum majorana L. by means of SCAR-PCR in commercial samples of Mediterranean oregano. Food Control, 22(3-4), 542-548.Moore, J. C., Spink, J., \& Lipp, M. (2012). Development and Application of a Database of Food Ingredient Fraud and Economically Motivated Adulteration from 1980 to 2010. Journal of Food Science, 77(4), R118-R126.

Moore, J. C., Spink, J., \& Lipp, M. (2012). Development and Application of a Database of Food Ingredient Fraud and Economically Motivated Adulteration from 1980 to 2010. Journal of Food Science, 77(4), R118-R126. 
Parveen, I., Gafner, S., Techen, N., Murch, S. J., \& Khan, I. A. (2016). DNA Barcoding for the Identification of Botanicals in Herbal Medicine and Dietary Supplements: Strengths and Limitations. Planta Medica, 82(14), 1225-1235.

Sanzini, E., Badea, M., Dos Santos, A., Restani, P., \& Sievers, H. (2011). Quality control of plant food supplements. Food \& Function, 2(12), 740-746.Kress, W. J., Wurdack, K. J., Zimmer, E. A., Weigt, L. A., \& Janzen, D. H. (2005). Use of DNA barcodes to identify flowering plants. Proc Natl Acad Sci US A, 102(23), 8369-8374.

Sumner, L. W., Amberg, A., Barrett, D., Beale, M. H., Beger, R., Daykin, C. A., Fan, T. W. M., Fiehn, O., Goodacre, R., Griffin, J. L., Hankemeier, T., Hardy, N., Harnly, J., Higashi, R., Kopka, J., Lane, A. N., Lindon, J. C., Marriott, P., Nicholls, A. W., Reily, M. D., Thaden, J. J., \& Viant, M. R. (2007). Proposed minimum reporting standards for chemical analysis. Metabolomics, 3(3), 211-221.

Taberlet, P., Coissac, E., Pompanon, F., Gielly, L., Miquel, C., Valentini, A., Vermat, T., Corthier, G., Brochmann, C., \& Willerslev, E. (2007). Power and limitations of the chloroplast trnL (UAA) intron for plant DNA barcoding. Nucleic Acids Research, 35(3).

United Nations (1992). United nations' Convetion on Biological Diversity, 1992, https://www.cbd.int/doc/legal/cbd-en.pdf Accessed 05/01/2017

U.S. Pharmacopeial Convention (2016). USP's Food Fraud Database. https://www.foodfraud.org/

Xie, P. S., Chen, S. B., Liang, Y. Z., Wang, X. H., Tian, R. T., \& Upton, R. (2006). Chromatographic fingerprint analysis - a rational approach for quality assessment of traditional Chinese herbal medicine. Journal of Chromatography A, 1112(1-2), 171-180.

Yu, J., Xue, J.H., Zhou, S.L. (2011). New universal matK primers for DNA barcoding angiosperms. Journal of Systematics and Evolution 49 (3): 176-181.

Yuan, J. J., Wang, C. Z., Chen, H. X., Ye, J. Z., \& Zhou, H. (2016). Identification and Detection of Adulterated Camellia Oleifera Abel. Oils by Near Infrared Transmittance Spectroscopy. International Journal of Food Properties, 19(2), 300-313.

\section{Captions:}

Figure 1. Monitored transitions of the selected adulterant markers during LC-MS/MS analysis with the respective chromatographic traces (presented in the respective row). Markers and transitions with asterisk were employed as quantifiers.

Figure 2: Qualitative PCA and Quantitative PLS-R models for oregano Origanum vulgare and Origanum onites adulterated with olive leaves and Myrtaceae leaves based on FTIR data.

Figure 3. Correlation of quantitation results between FTIR screening method and LC-MS/MS targeted method for olive (a, b) and Myrtaceae leaves (c) results. Graphs a) and b) represent two differing sample sets i.e. a) include all of the adulterated samples above FTIR LOD $(n=51)$ while $b)$ only samples below $90 \%$ adulteration level.

Figure 4. a) Composition of the adulteration in the assessed samples (sum of adulteration confirmed by LC-MS/MS and FTIR for cistus). b) Percent total adulteration distribution in positive samples (whiskers 5-95 percentile). 
Table 1. Results of biodiversity assessment based on measured \% adulteration in 21 extracts of varying mixtures of oregano $(n=7)$ and either olive $(n=7)$ or Myrtaceae leaves $(n=6)$ samples at adulteration level of $10 \%$. ${ }^{\text {a }}$ One sample was rejected due to analyst's random error during the extraction.

\begin{tabular}{|c|c|c|c|c|c|c|c|c|}
\hline \multirow{2}{*}{$\begin{array}{c}\begin{array}{c}\text { Oregano } \\
\text { leaves }\end{array} \\
\begin{array}{c}\text { Sample } \\
\#\end{array}\end{array}$} & \multicolumn{4}{|c|}{ Olive leaves } & \multicolumn{4}{|c|}{ Myrtaceae leaves } \\
\hline & $\begin{array}{c}\text { Sample } \\
\#\end{array}$ & $\begin{array}{c}\text { Measured } \\
\text { adulteration } \\
{[\%]}\end{array}$ & $\begin{array}{c}\text { Repeatability } \\
{[\mathrm{CV}, \%]}\end{array}$ & $\begin{array}{c}\text { Accuracy } \\
{[\%]}\end{array}$ & $\begin{array}{c}\text { Sample } \\
\#\end{array}$ & $\begin{array}{c}\text { Measured } \\
\text { adulteration } \\
{[\%]^{\mathrm{a}}}\end{array}$ & $\begin{array}{c}\text { Repeatability } \\
{[\mathrm{CV}, \%]}\end{array}$ & $\begin{array}{c}\text { Accuracy } \\
{[\%]}\end{array}$ \\
\hline \multirow{7}{*}{24} & & 10.5 & \multirow{7}{*}{7} & \multirow{7}{*}{92} & & 10.4 & \multirow{7}{*}{3} & \multirow{7}{*}{105} \\
\hline & & 9.0 & & & & 10.5 & & \\
\hline & & 9.2 & & & & 10.6 & & \\
\hline & CRM & 9.4 & & & 4 & 10.8 & & \\
\hline & & 8.9 & & & & 10.9 & & \\
\hline & & 9.1 & & & & 10.1 & & \\
\hline & & 8.7 & & & & & & \\
\hline \multirow{7}{*}{24} & 1 & 13.3 & \multirow{7}{*}{13} & \multirow{7}{*}{109} & 3 & 14.5 & \multirow{7}{*}{18} & \multirow{7}{*}{110} \\
\hline & 2 & 11.5 & & & 4 & 10.2 & & \\
\hline & 21 & 11.3 & & & 19 & 11.8 & & \\
\hline & 25 & 11.1 & & & 27 & 10.9 & & \\
\hline & 90 & 10.8 & & & 89 & 10.5 & & \\
\hline & 123 & 8.6 & & & 125 & 7.8 & & \\
\hline & CRM & 10.0 & & & 27 & 11.5 & & \\
\hline 10 & \multirow{7}{*}{ CRM } & 12.0 & \multirow{7}{*}{17} & \multirow{7}{*}{113} & & 7.9 & \multirow{7}{*}{13} & \multirow{7}{*}{100} \\
\hline 11 & & 14.5 & & & & 12.2 & & \\
\hline 12 & & 10.4 & & & & 10.7 & & \\
\hline 24 & & 10.8 & & & 4 & 9.5 & & \\
\hline 28 & & 11.4 & & & & 8.6 & & \\
\hline 31 & & 8.9 & & & & 9.3 & & \\
\hline CRM & & 13.6 & & & & 9.5 & & \\
\hline
\end{tabular}


Table 2. Results of WRL assessment based on measured \% adulteration in three mixtures, all containing both Myrtaceae and olive leaves, each extracted 6 times, on three different days $(n=54)$.

\begin{tabular}{ccccccc}
\hline Adulterant & $\begin{array}{c}\text { Adulteration } \\
\text { level } \\
{[\%]}\end{array}$ & $\begin{array}{c}\text { Reproducibility } \\
{[\mathrm{CV}, \%]}\end{array}$ & $\begin{array}{c}\text { Accuracy } \\
{[\%]}\end{array}$ & $\begin{array}{c}\mathrm{CC} \alpha \\
{[\%]}\end{array}$ & $\begin{array}{c}\text { CC } \beta \\
{[\%]}\end{array}$ & $\begin{array}{c}\text { Experimental } \\
\text { CC } \beta \\
{[\%]}\end{array}$ \\
\hline $\begin{array}{c}\text { Olive } \\
\text { leaves } \\
(\mathrm{n}=54)\end{array}$ & $10 \%$ & 8.3 & 138 & & & \\
\hline $\begin{array}{c}\text { Myrtaceae } \\
\text { leaves } \\
(\mathrm{n}=54)\end{array}$ & $30 \%$ & 7.5 & 107 & 1.27 & 2.16 & 1.76 \\
\hline
\end{tabular}




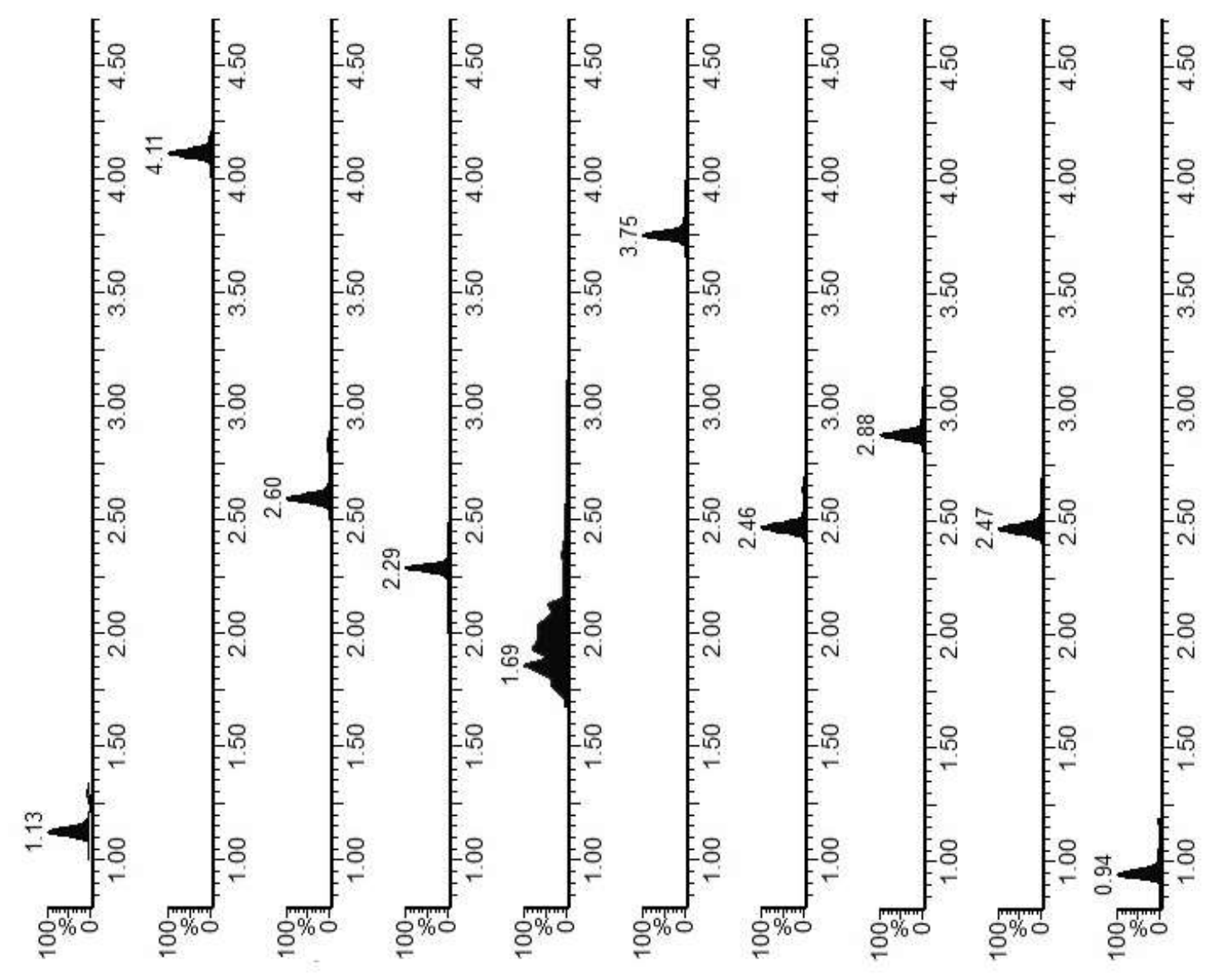

\begin{tabular}{|c|c|c|c|c|c|c|c|c|c|c|}
\hline च्ञ & ' & & + & . & + & + & + & + & . & ' \\
\hline 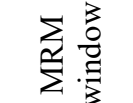 & $r$ & 은 & $\nabla$ & 6 & in & $m$ & $N$ & - & $a$ & $\infty$ \\
\hline 된 $\geq$ & 규 ন & 우 웃 & 이 సి గ & 우 तి ㄱ & ㅇ & in & 고 응 & 구응 & i & 요 तి \\
\hline 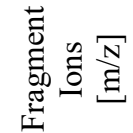 & 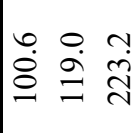 & 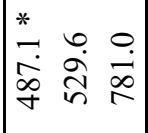 & 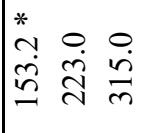 & 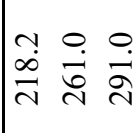 & 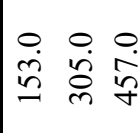 & 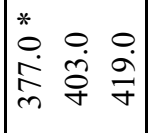 & 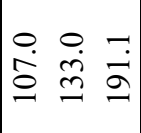 & 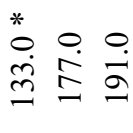 & $\left|\begin{array}{ccc}0 & 0 & 0 \\
\infty & \infty & 0 \\
= & \infty & \stackrel{n}{*}\end{array}\right|$ & 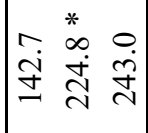 \\
\hline 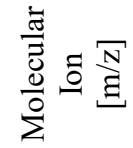 & 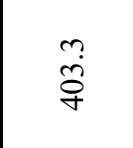 & 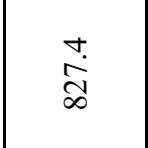 & $\begin{array}{l}\stackrel{+}{4} \\
\tilde{n} \\
\tilde{n}\end{array}$ & 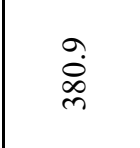 & $\begin{array}{l}\stackrel{0}{0} \\
\hat{0}\end{array}$ & $\begin{array}{l}\stackrel{0}{0} \\
\text { in }\end{array}$ & 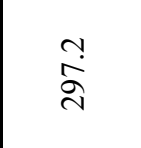 & $\overrightarrow{\vec{a}}$ & $\stackrel{m}{\stackrel{n}{f}}$ & $\begin{array}{l}\infty \\
\stackrel{0}{0} \\
\stackrel{n}{+}\end{array}$ \\
\hline 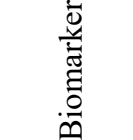 & 0 & * & $\stackrel{*}{\Sigma}$ & $\dot{\Sigma}$ & $\tilde{\tilde{N}}$ & $\stackrel{*}{*}$ & $\bar{\Xi}$ & $\begin{array}{l}* \\
\stackrel{I}{I}\end{array}$ & $\dot{1}$ & 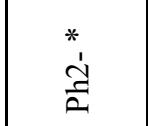 \\
\hline 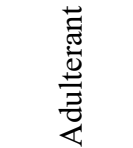 & & 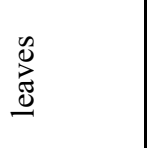 & $\sum_{\Sigma}^{0}$ & 岂 & & 节 & & 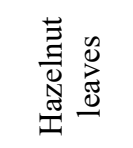 & & $\frac{.00}{\frac{0}{a}}$ \\
\hline
\end{tabular}




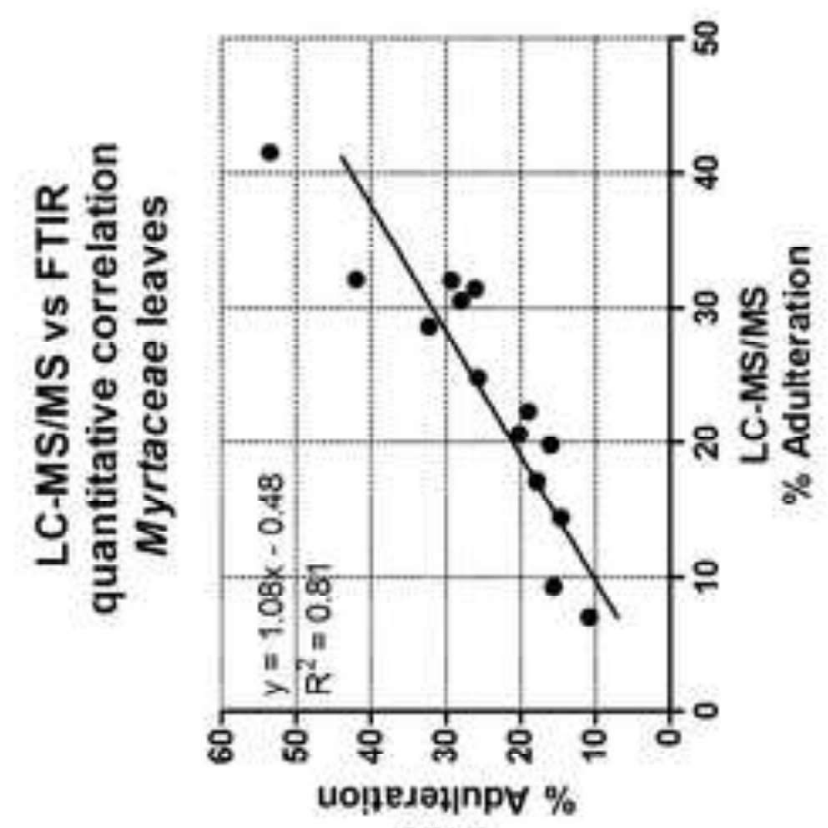

비너
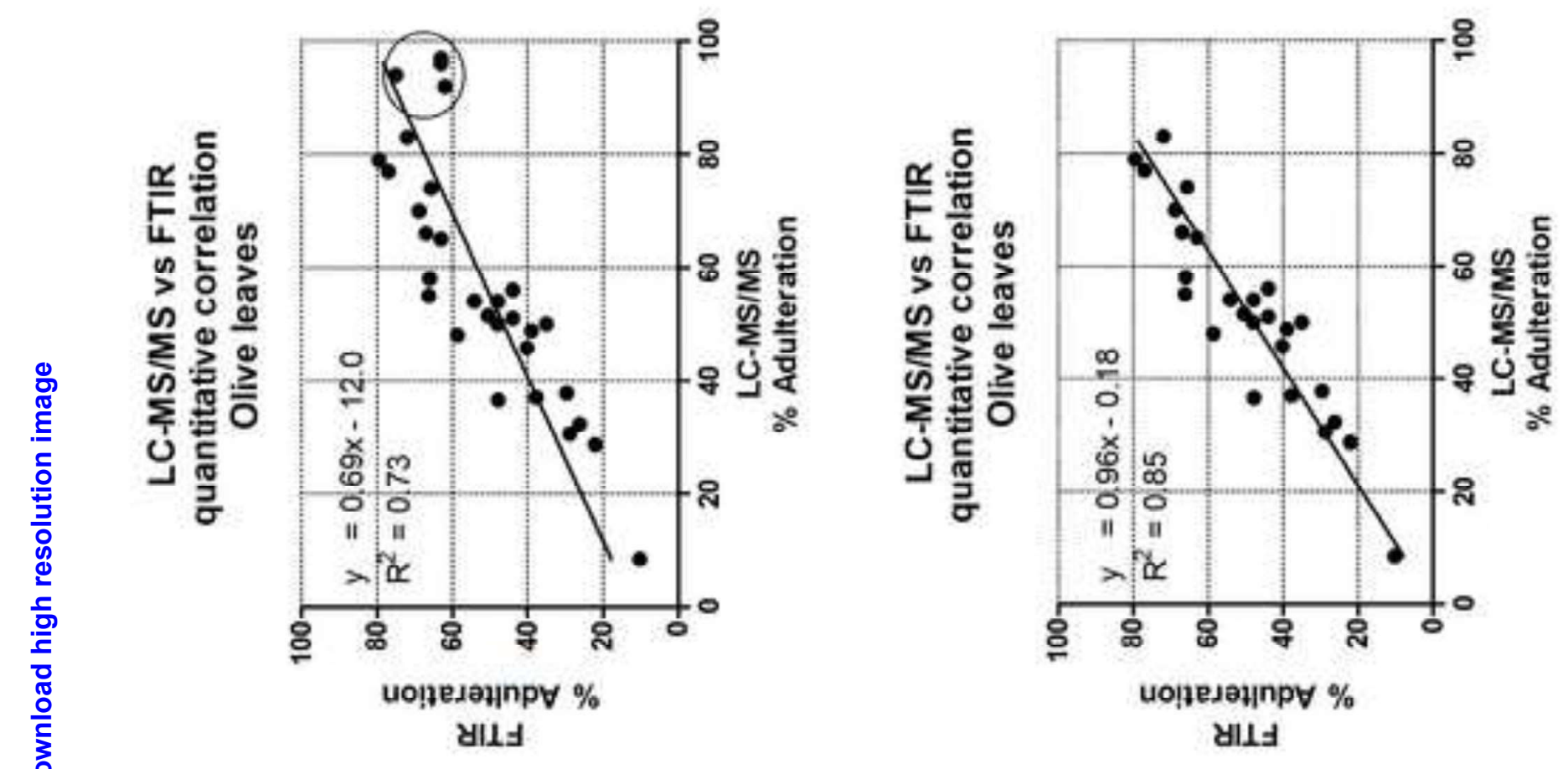


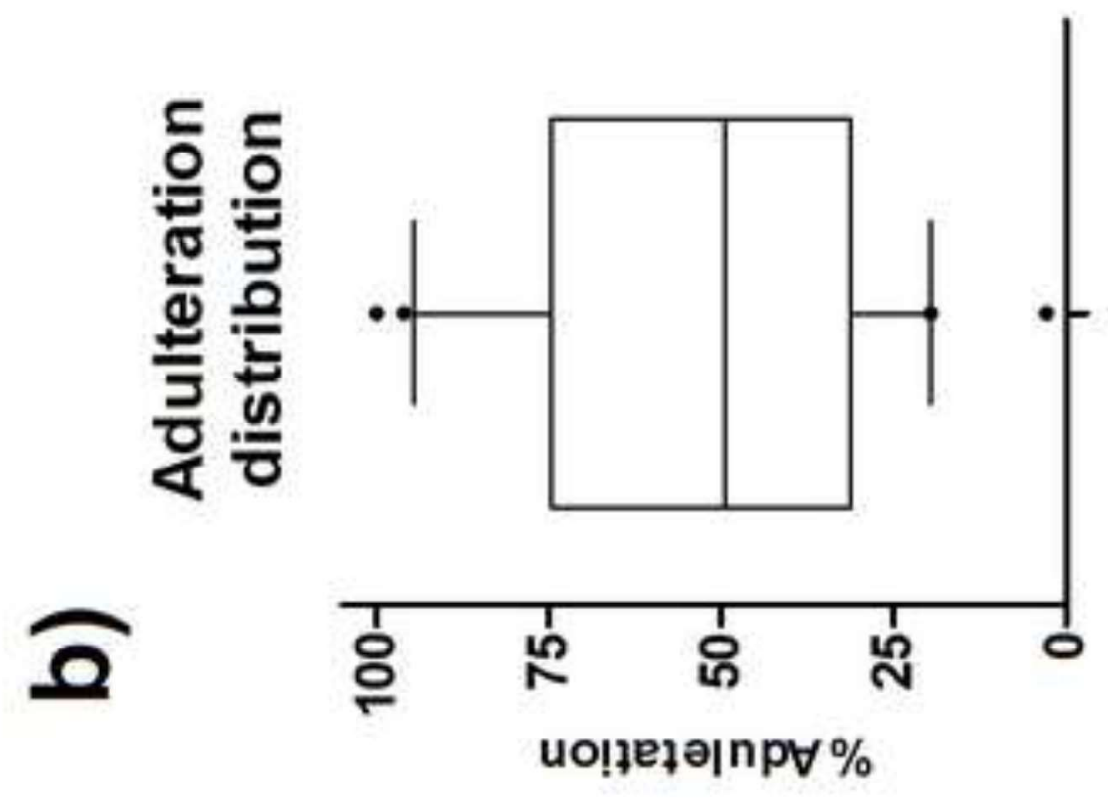

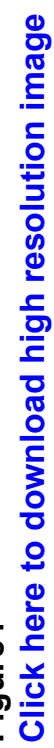

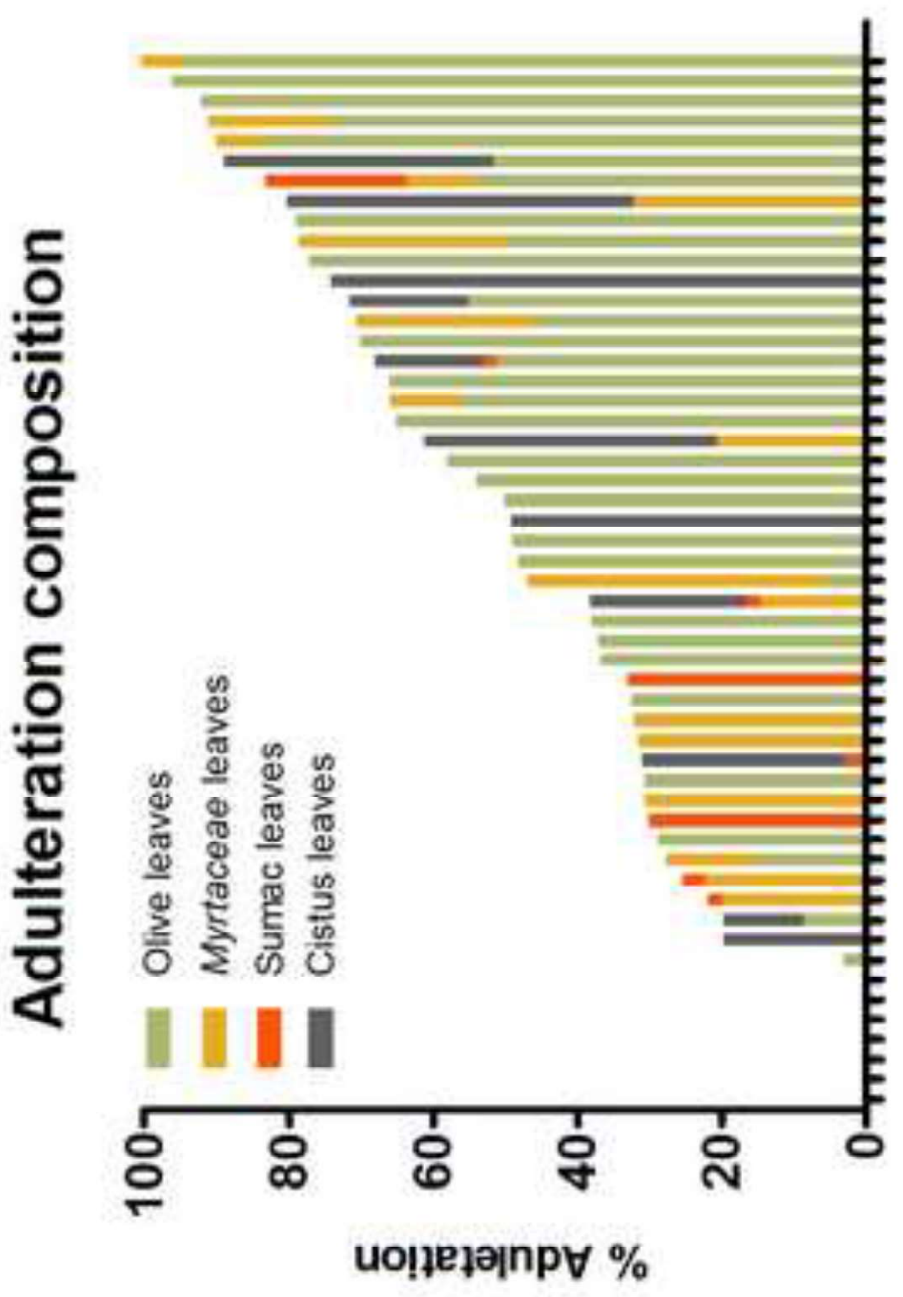


Click here to download Supplementary Material: SUPPLEMENTARY MATERIAL.docx 
- A novel analytical platform, allowing for detection, confirmation and quantitation of herb adulteration in 30min, has been developed.

- A confirmatory, LC-MS/MS method, based on biomarker discover, has been successfully validated.

- The comparison of the LC-MS/MS method and a screening FTIR method, revealed good correlation of adulteration quantitation.

- A survey of suspected samples revealed high incident and levels of oregano adulteration. 